\title{
HIGH POWER NANOCRYSTALLINE DIAMOND RF MEMS- A COMBINED LOOK AT MECHANICAL AND MICROWAVE PROPERTIES
}

\author{
S.Balachandran $^{1}$, J.Kusterer ${ }^{2}$, D.Maeir ${ }^{2}$, M.Dipalo ${ }^{2}$, T.Weller ${ }^{1 *}$, E.Kohn ${ }^{2}$ \\ ${ }^{1}$ Department of Electrical Engineering, University of South Florida, Tampa, FL, USA \\ ${ }^{2}$ Department of Electron Devices and Circuits, University of Ulm, Ulm, Germany
}

\section{Introduction}

Radio frequency micro-electro mechanical systems (RF-MEMS) technology has been growing rapidly in the last few years and has found a multitude of applications in the automotive, defence and communication industries. Due to their outstanding properties and excellent performance at high frequencies, RF MEMS devices have started replacing their solid state counterparts in filters [1], phase shifters [2] and antennas [3]. According to a 2006 market study, the global market for MEMS, with RF-MEMS being a major part will reach $\$ 12$ billion by 2010 [4].

Many RF-MEMS devices have been successfully implemented on different substrates with different actuation schemes [5]. Although they are small in size and exhibit low parasitic losses, the monolithic configuration confines the device to a substrate common to the entire system. Furthermore, power handling [6] capabilities of RF-MEMS devices have been limited due to using all metal structures in the devices. Diamond has long been used in active devices such as FETS for high power electronics [7]. Also its stability under high temperature $\left(1000^{\circ} \mathrm{C}\right)$ makes it a very good candidate for realizing reliable, high power and temperature stable RF-MEMS and microwave devices.

In this paper, a compressively stressed nanocrystalline diamond (NCD) actuator utilizing a thermal actuation scheme is presented. The growth chemistry of NCD films along with the mechanical properties of diamond are discussed in detail. Stress engineering is performed to realize compressively stressed diamond films for RF-MEMS applications. A NCD based bi-stable actuator is designed and fabricated on a low resistive silicon substrate. Tunable switches are implemented in CPW and microstrip topologies and small signal measurements are performed in the frequency range of 5-30 GHz. High power measurements are performed in the power spectrum of 24-47 $\mathrm{dBm}$ on the switches integrated in the microstrip topology.

\section{Diamond Growth \& Mechanical Properties}

Thin NCD films are grown through a chemical vapor deposition (CVD) process wherein the growth occurs by the decomposition of carbon containing precursor molecules (typically methane) in either a pure hydrogen, or 
hydrogen and argon environment. NCD growth is done through a thermal (hot filament) [8], plasma (microwave or RF) [9] activation or use of a combustion flame (oxyacetylene). Of the three, hot filament and microwave plasma methodologies are the most popular techniques used for thin film diamond growth. Prior to diamond growth, the wafer needs to go through a seeding step which aids in the growth of the thin film. In this work, thin film diamond films are grown in the HFCVD technique using bias enhanced nucleation (BEN) as the seeding method. The HFCVD technique is based on the heating of metal filaments up to $2000^{\circ}-2200^{\circ} \mathrm{C}$ in order to break molecular hydrogen and carbon compounds and to form $\mathrm{C}_{\mathrm{y}} \mathrm{H}_{\mathrm{x}}$ free radicals; these free radicals move toward the substrate by a temperature gradient. On the substrate surface both diamond $\left(\mathrm{sp}^{3}\right)$ and graphite $\left(\mathrm{sp}^{2}\right)$ bonds are formed; the graphite bonds are however etched by atomic hydrogen allowing diamond growth on the substrate surface. Figure 1 shows an SEM image of NCD film grown in an HFCVD reactor.

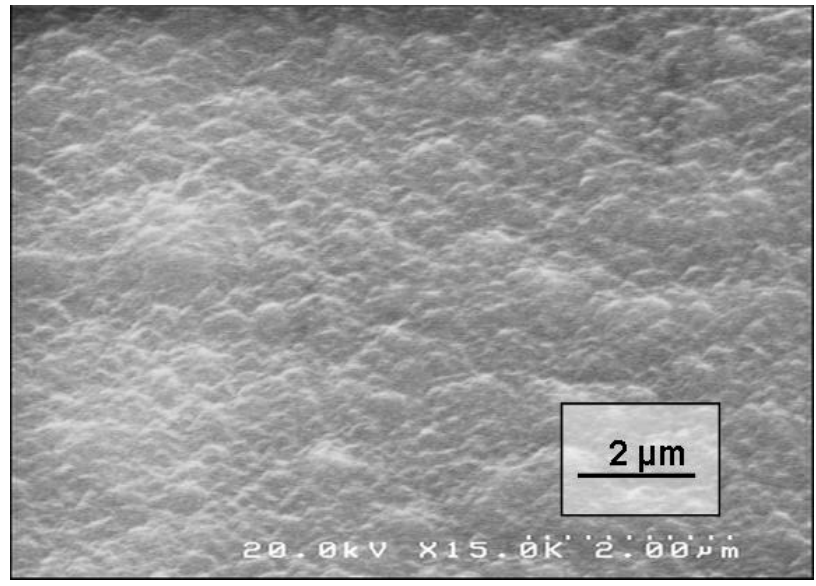

Figure 1- SEM image of the NCD film grown by HFCVD technique

Apart from its application in the area of electrochemistry and optics, nanocrystalline diamond due to its superior mechanical properties is an ideal candidate for MEMS technology. To validate this claim Young's modulus, intrinsic stress and fracture strength of the NCD film are measured. Young's modulus gives a good insight into the stiffness of a material and its spring constant. In MEMS actuators, it is best to have materials with high Young's modulus for reliability purposes. Comparing other materials, higher resonant frequency and higher switch-off time can be realized by using NCD. Furthermore, because of such high Young's modulus, thin yet stiff beams can be fabricated which allows the overall down-scaling of the device.

In this work, Young's modulus is measured using a non-destructive technique. The first order mechanical resonant frequency of a micromachined cantilever is measured by stimulating the structure using a piezo oscillator. The resonant frequency of the cantilever is dependent on the thickness $(t)$ of the diamond film, length of the cantilever (1), density of the material $(\rho)$ and the Young's modulus $(\mathrm{E})$ of the thin film. This is mentioned in equation 1 [10]. 


$$
F_{\mathrm{Re} s}=0.16 * \sqrt{\frac{E}{\rho}} * \frac{t}{l^{2}}
$$

For a $1.2 \mu \mathrm{m}$ thick, 100 micron long cantilever structure, Young's modulus was around $1020 \mathrm{GPa}$. In comparison, the Young's modulus for silicon would be in the range of 150-160 GPa.

With the increasing use of micromachining techniques for the fabrication of MEMS devices it is important to understand stress conditions within the thin films. Stress in NCD films can be changed widely $(-500 \mathrm{MPa}$ to $+700 \mathrm{MPa})$ by controlling the growth parameters that include pressure, substrate temperature and gas ratio involved. There are various techniques which are available to measure the compressive and tensile stress in the film [11]. In this work, rotation tips technique is used to measure the compressive stress in the NCD film. In this technique, the strain is converted into a rotation angle which is directly proportional to the strain caused in the material. Although this technique can be used for measuring both kinds of stresses (tensile and compressive), our application demands compressively stressed NCD structures. Under varying growth conditions, compressive stress ranging from $140 \mathrm{MPa}$ to $557 \mathrm{MPa}$ for a $1.2 \mu \mathrm{m}$ thick film is measured. Figure 2(a) and 2(b) shows the SEM image of the rotation tip structures used for measuring the stress.

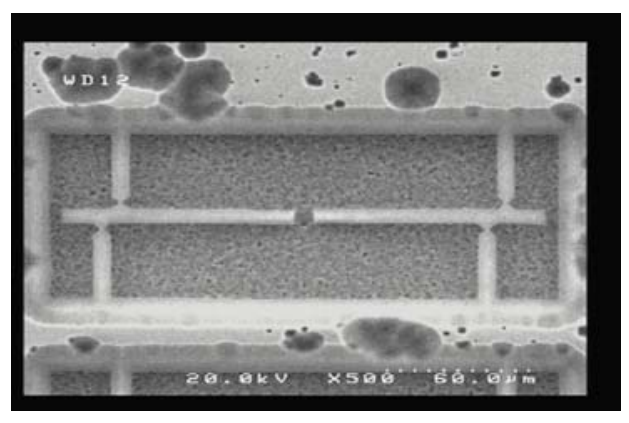

(A)

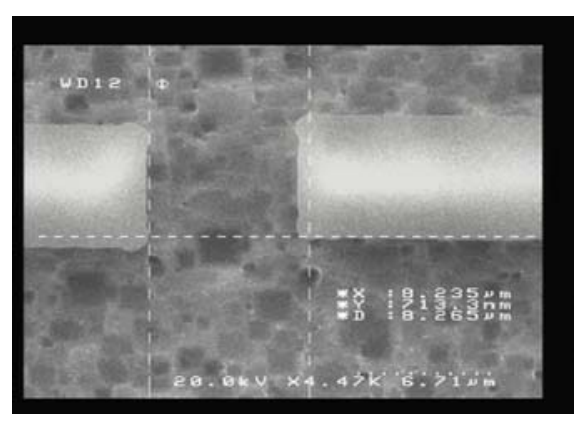

(B)

Figure 2- Releases structures used for measuring the intrinsic stress in NCD films

\section{Actuator Design and Operation}

The actuator comprises of a diamond beam anchored at both sides in the silicon frame with thermal switching elements. The bridges are compressively pre-stressed during the growth process thereby realizing a buckled beam. The buckled diamond bridges are $1200 \mu \mathrm{m}$ long and $300 \mu \mathrm{m}$ wide. Upon release, the bridges are bi-stable in nature and can switch between the two quasi-stable positions upon actuation. The use of NCD film enables little to no stress profile due to the homogenous morphology (grain size and distribution) in the vertical direction. The bridges are thermally actuated using a bi-metal actuation scheme. [12]. Compared to electrostatic actuation, thermal actuation has the advantage of having a lower actuation voltage and a higher contact 
force. The main drawback of thermal actuation scheme is the static power consumption which can be avoided by using a bi-stable layout.

The basic device consists of a bridge made of doped diamond. A second metal layer (copper) is deposited on top of the diamond bridge. The difference in coefficient of thermal expansion between the two materials, $0.8 * 10^{-6} / \mathrm{K}$ for diamond and $13 * 10^{-6} / \mathrm{K}$ for copper causes the bending moment and resistive heating of the doped areas forces a bending of the beam and hence switching into the actuated state. The pull-in voltage (and current) to switch the bridge depends on the geometry of the diamond heating elements and is in the range of 2-6 volts and 5-20 mA. The design of the diamond actuator is shown in Figure 3. In the first iteration of the design, nickel was chosen to the bi-metal [13]. Apart from the higher coefficient of thermal expansion, the number of processing steps can be reduced by using copper. Gold wirings are included in the design to provide a DC electrical path to the contact pad at the center of the diamond bridge. The contact pads are $100 \mu \mathrm{m} \times 100 \mu \mathrm{m}$ in dimension.

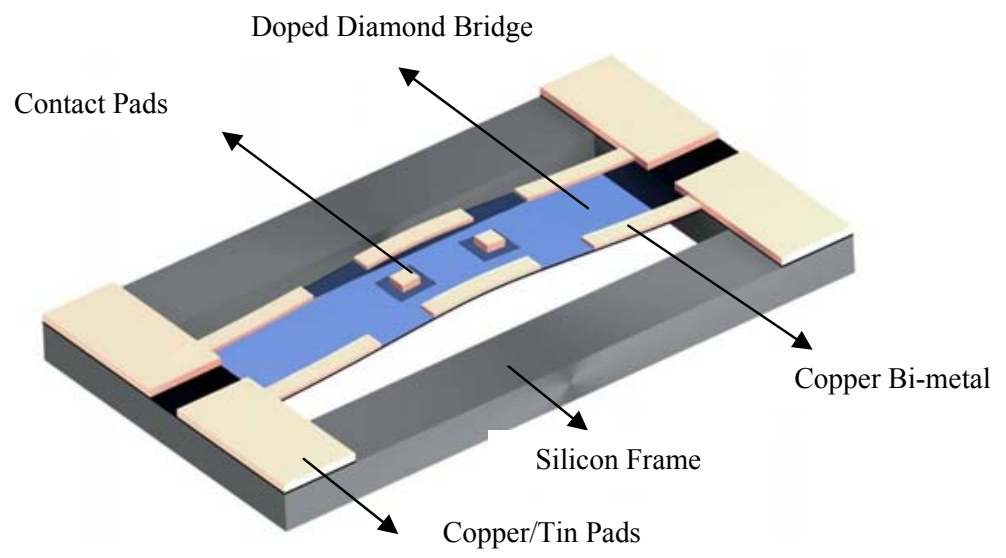

Figure 3- Design of the compressively stressed NCD diamond actuator

The buckled diamond bridges are bi-stable in operation. Buckling is achieved during the diamond growth process wherein intentional compressive stress is introduced. This stress during growth results in two stable positions of the actuator; one state with a deflection of the beam centre downwards and a second state with a stable deflection upwards. During actuation, a voltage pulse is applied between the two parallel copper stripes contacting the doped diamond layer, resulting in current flow across the diamond resistor and thereby generating the temperature needed for the bending moment.

\section{Fabrication and Integration of the Actuator}

The diamond bridges are fabricated on a 2 inch low resistive silicon substrate which is $500 \mu \mathrm{m}$ thick. The fabrication steps are as follows:

- Prior to growth, the silicon wafer is seeded using a bias enhanced nucleation (BEN) technique wherein diamond clusters are formed. A 2 $\mu \mathrm{m}$ thick boron doped diamond layer is grown on top of the seeding layer using a hot filament chemical vapor deposition (HFCVD) reactor. 
- Intrinsic diamond is selectively grown using a $\mathrm{SiO}_{2}$ hard mask. This $4000 \mathrm{~A}^{0}$ thick diamond is used for electrical isolation while the switch is actuated.

- Copper bi-metal pads are electroplated to a thickness of $1 \mu \mathrm{m}$. Before electroplating, a $\mathrm{Cr} / \mathrm{Au}$ seed layer of $1000 \mathrm{~A}^{0}$ thickness is deposited using a thermal evaporator.

- Copper pads which are used for integration are electroplated to a thickness of $12 \mu \mathrm{m}$. The RF contact areas are also formed by electroplating in this step.

- To avoid oxidation, $500 \mathrm{~A}^{0}$ of platinum is patterned over the copper contact pads using lift-off technique

- Diamond bridges are patterned by oxygen based RIE with titanium as the hard mask

- Finally, using patterned silicon dioxide as a backside hard mask, diamond structures are released from the silicon wafer through a "Bosch process" resulting in a free standing diamond bridge that is embedded in a silicon frame.

Prior to this design, the diamond actuator was fabricated in which the bridge was composed of intrinsic diamond with areas of selectively grown doped diamond. The doped diamond bridge was chosen so that resistance of the heaters can be lower without actually making the bridge thicker and stiffer. Figure 4(a) and 4(b) shows the front view and the back view of the fabricated diamond actuator. The overall size of the entire chip is $1600 \mu \mathrm{m}$ long and $900 \mu \mathrm{m}$ wide.

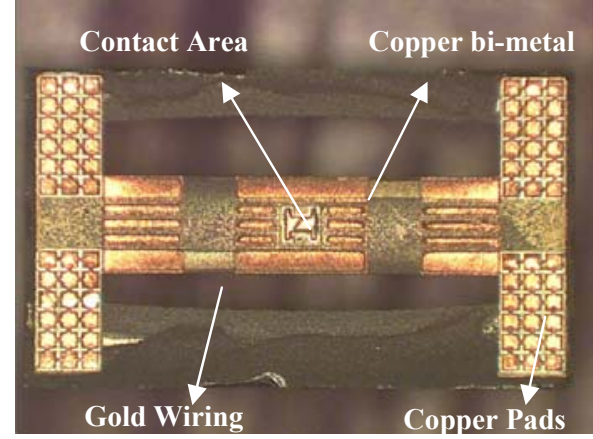

(A)

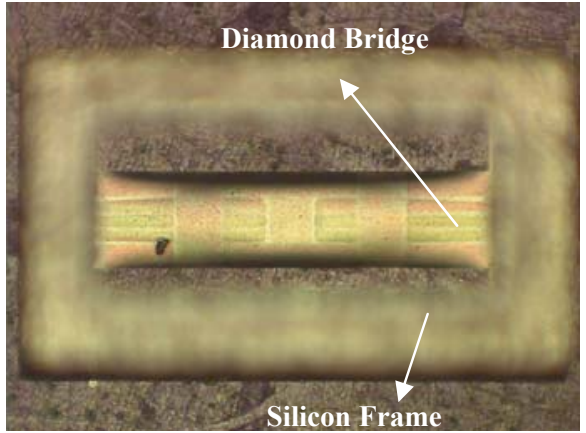

(B)

Figure 4- Top view of the fabricated diamond actuator (A) front view (B) back view of the actuator with the silicon frame

Being monolithic in design, this diamond actuator is substrate independent and can be integrated to any microwave substrate depending on the application. For the first set of results these actuators were flip chip bonded to an alumina substrate using a $\mathrm{Cu} / \mathrm{Sn}$ solid liquid inter-diffusion (SOLID) process [14]. Figure 5 shows the integration process of the diamond actuator to the host substrate.

In addition to the copper pads in the actuator frame, copper pads along with tin should be included in the host substrate for integration. Solid-liquid 
interdiffusion occurs between the two phases, resulting in a phase transformation of the liquid component to a higher melting point material, which is strong enough to serve as a bond and withstand elevated temperatures. Copper-Tin has been considered a SOLID couple where tin acts a melting phase and copper as a solid phase. While integrating, the copper pads on the actuator are kept on top of the copper-tin stack and heated till $250^{\circ} \mathrm{C}$ (melting point of tin). During the diffusion process one of the intermetallic compounds forms the bond between the two structures and is stable till $600^{\circ} \mathrm{C}$. The alignment is carried out in a flip-chip bonding test setup wherein an accuracy of \pm 5 microns is achieved during the integration (Figure 5). Heating for the solder process is supplied from the actuator part to prevent alloying of the pads that are located on the host substrate.

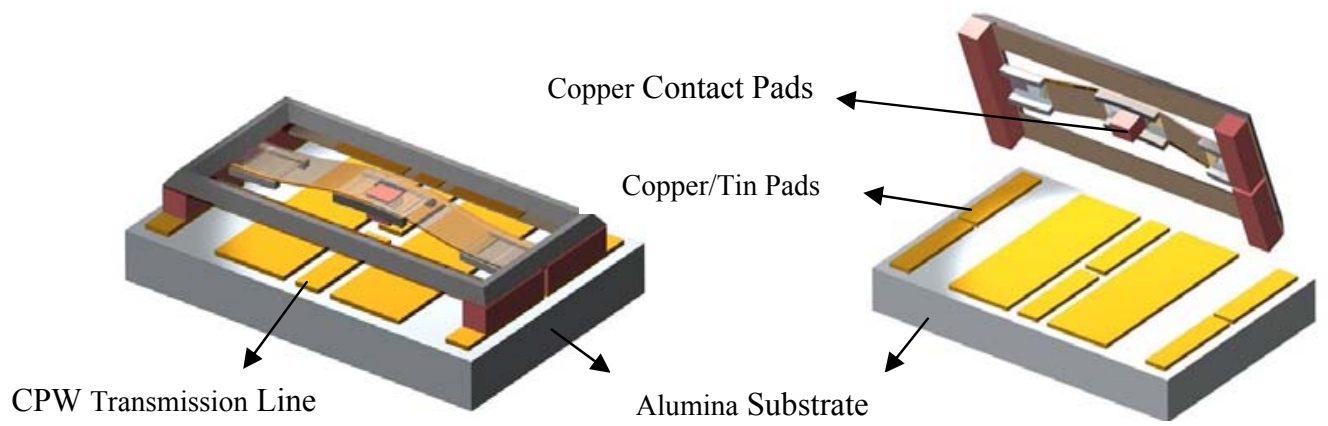

Figure 5- Diamond actuator integrated onto the host alumina substrate using the solid liquid interdiffusion (SOLID) process

\section{Microwave Characterization}

The diamond actuators are used to realize RF-MEMS DC contact type switches in CPW and microstrip topologies. The CPW transmission lines are designed on a $650 \mu \mathrm{m}$ thick alumina substrate $\left(\varepsilon_{\mathrm{r}}=9.9, \tan \delta=0.0002\right)$. The transmission lines are $3000 \mu \mathrm{m}$ long with a center conductor width (W) of $100 \mu \mathrm{m}$ and slot width $(\mathrm{G})$ of $50 \mu \mathrm{m}$. The center conductor of these lines is purposefully interrupted in the middle resulting in two transmission lines which are $1475 \mu \mathrm{m}$ long. During actuation, the contact pad in the diamond bridge closes this gap resulting in regular transmission line. Small signal measurements are done in the frequency span of 1-30 GHz using an Anritsu Lightning VNA. A bias tee was used to protect the VNA test ports from DC current. Before measuring the structures a probe-tip SOLT calibration is performed on a commercial GGB CS-9 calibration. The diamond bridges are thermally actuated at 2 volts wherein the platinum coated copper pad makes contact with the CPW line. Figure 6(a) and 6(b) shows the $S_{11}$ and $S_{21}$ of the transmission line in the non-actuated and actuated state respectively. 

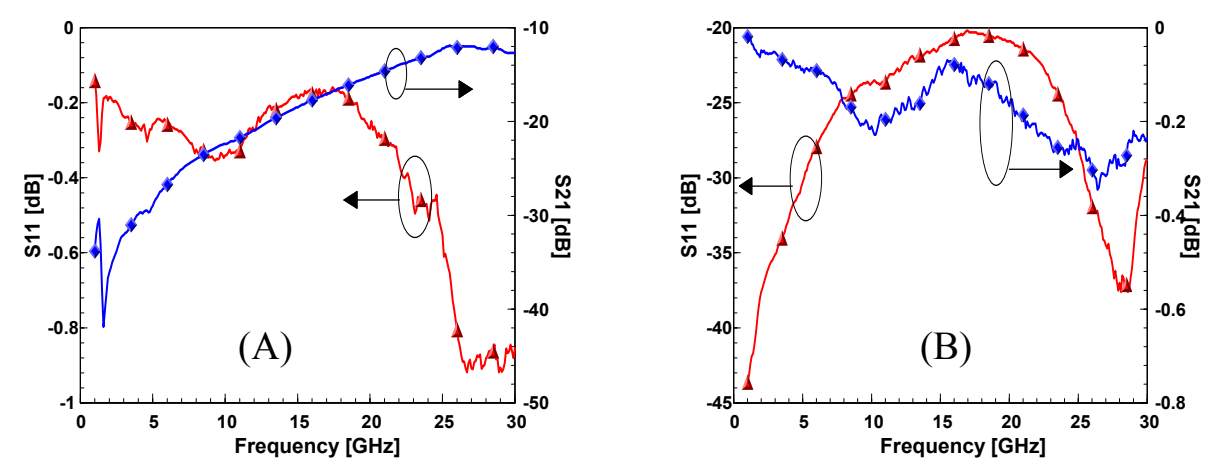

Figure 6- Insertion and Return loss of the CPW switch in the (A) Nonactuated state and (B) Actuated state

Apart from the CPW switch, the diamond actuators were used to realize tunable inductors and switches in CPW and microstrip topologies respectively [15]. Furthermore, high power measurements are carried out at $2.1 \mathrm{GHz}$ in the power range of $24-47 \mathrm{dBm}$. The power density at the overlapping areas is estimated to be in the range of $18 \mathrm{KW} / \mathrm{mm}^{2}$. Insertion loss of $0.2-0.3 \mathrm{~dB}$ was measured in the entire power spectrum with the device still operational after the test. Measurements were made for more than one instance for repeatability and there was no significant difference in the insertion loss. The diamond bridge was stable at such power with little or no damage due to heating of the structure. Diamond which also is an excellent conductor of heat acts a good heat sink during measurement.

\section{Conclusion}

In this paper, thermally actuated nanocrystalline diamond bridges are presented. Intrinsic and boron doped NCD films have been grown on low resistive silicon wafer. Young's modulus of $1020 \mathrm{GPa}$ was measured using a non-destructive technique. The films were intentionally grown with built in compressive stress and the measured values were in the range of 140-560 $\mathrm{MPa}$. Design, fabrication and integration of the compressively stressed bistable actuators are presented in detail.

The actuators were used to realize switches in CPW and microstrip topologies. Measured s-parameters show a return loss of $20 \mathrm{~dB}$ and insertion loss of 0.2 $\mathrm{dB}$ at $20 \mathrm{GHz}$. High power measurements were performed in the range of 24$47 \mathrm{dBm}$ at $2.1 \mathrm{GHz}$ with an insertion loss of $0.2-0.3 \mathrm{~dB}$ in the entire range.

\section{Acknowledgement}

The authors would like to thank Dr. Wolfgang Ebert from University of Ulm for his support in diamond growth, Dr. Ulrich Heinle from MicroGaN GmbH for his assistance in deep silicon etching and Mr. Rick Connick from Modelithics Inc. for his assistance in the high power measurement. This work was supported by The USA National Science Foundation, grant number ECS0404137 and DFG (German Research Foundation). 


\section{References}

[1] J.Brank et al., "RF MEMS-based tunable filters", International Journal RF Microwave CAE, Vol.11, pp. 276-284, September 2001.

[2] N.S.Barker et al., "Distributed MEMS true-time delay phase shifters and wide band switches", IEEE Transactions on Microwave Theory and Techniques, Vol. 46, No. 11, November 1998.

[3] C.W.Baek et al., "2D mechanical beam steering antenna fabricated using MEMS technology", IEEE MTT-S International Microwave Symposium, pp. 211-214, Phoenix, AZ, June 2001

[4] J.Bryzek et al., "Marvelous MEMS", IEEE Circuits and Devices Magazine, March-April 2006.

[5] G.M.Rebeiz, "RF MEMS- Theory, Design and Technology", John Wiley and Sons Publication, 2003.

[6] D.Peroulis et al., "RF MEMS switches with enhanced power handling capabilities", IEEE Transactions on Microwave Theory and Techniques, Vol. 52, No. 11, January 2004,

[7] A.Aleksov et al., "First diamond FET RF power measurement on diamond quasi-substrate", 60 $6{ }^{\text {th }}$ Device Research Conference, June 2002.

[8] M.Amaral et al., "Growth rate improvements in the hot-filament CVD deposition of nanocrystalline diamond", Diamond and Related Materials, November 2006.

[9] M.Miyake et al., "Characteristics of nano-crystalline diamond films prepared in $\mathrm{Ar} / \mathrm{H}_{2} / \mathrm{CH}_{4}$ microwave plasma", Diamond and Related Materials, March 2007.

[10] J.M.Gere et al., "Mechanics of Materials", $4^{\text {th }}$ edition, PWS Publishing Company, Boston, 1997.

[11] B.P. Van Drieenhuzen et al., "Comparison of techniques for measuring both compressive and tensile stress in thin films", Sensors and Actuators A, 37-38, 1993.

[12] P.Schmid et al., "Diamond switch using new thermal actuation scheme", Diamond and Related Materials, Vol. 12, Issues 3-7, March-July 2003.

[13] J.Kusterer et al., "Bi-stable micro actuator based on stress engineered nano-diamond", Diamond and Related Materials, Vol. 15, Issues 4-8, AprilAugust 2006.

[14] P.Benkart et al., "3D chip stack technology using through-chip interconnects", IEEE Design and Test of Computers, Vol. 22, Issue 6, NovDec 2005.

[15] S.Balachandran et al., " Thermally actuated nanocrystalline diamond micro-bridges for microwave and high power RF applications", IEEE MTT-S International Microwave Symposium, pp 367-370, Honolulu, HI, June 2007. 